Scientific Visualization, 2020, volume 12, number 2, pages 37 - 52, DOI: 10.26583/sv.12.2.04

\title{
The study of methods for analysis burning torch infrared images
}

\author{
I.A. Berg1,A, S.V. Porshnev 2,A,B \\ A Ural Federal University \\ B N.N. Krasovskii Institute of Mathematics and Mechanics of the Ural Branch of the Rus- \\ sian Academy of Sciences
}

${ }^{1}$ ORCID: 00oo-0003-4688-3964, berg77777@gmail.com

2 ORCID: 0000-0001-6884-9033

\begin{abstract}
The paper describes the results of the study of methods for analysis burning torch infrared images obtained by an infrared camera in the band of electromagnetic wavelengths of $1.5^{-}$ 5.1 $\mu \mathrm{m}$. It was shown that the known infrared image analysis methods cannot provide the quantitative parameters extraction that could describe combustion process. In addition, it was figured out that the known methods are time-consuming and cannot run in real time. As a result, nowadays the combustion control system that uses optical control of torch parameters in infrared band cannot be designed.

In our study we analyzed the pixels quantity distribution density in the range of [520,560] relative Celsius degrees on each frame of the initial infrared sequence of burning torch. It was shown that the pixels quantity distribution has the bimodal distribution law and can be described by three local extremes coordinates: two maximums and a minimum located between them. The pixels that have relative degrees values in the range from 520 degrees to the value of the minimum's abscissa and from the value of the minimum's abscissa to 560 degrees relatively form two separate zones on the burning torch visualization.

It was demonstrated that time-domain series constructed from frame-by-frame calculated local extremes coordinates of the $\mathrm{P}(\mathrm{T})$ distributions are stationary random sequences. This result allows to use these time-domain series as quantitative parameters of the torch combustion. It was shown that the local minimum's abscissa value of the $\mathrm{P}(\mathrm{T})$ distribution with a relative error of $2.8 \%$ is a constant value equal to 536.3 relative degrees. This allows to count the pixels quantity of each of the separate zones without using time-consuming Rosenblatt Parzen estimation and run data processing in real time.
\end{abstract}

Keywords: thermal imager, infrared band, torch combustion, data processing, informational parameters, Rosenblatt - Parzen estimation, stationarity.

\section{Introduction}

At present, the torch combustion systems that convert energy content of the fuel into the thermal energy are widely spread in various industry branches. Nonetheless, these systems have significant drawback, since the torch combustion is accompanied by formation of harmful combustion products and subsequent environmental pollution. Therefore, the reduction of harmful content (such as nitrogen oxides group $\mathrm{NO}_{\mathrm{x}}$ ) is the pressing problem. One of the possible approaches to harmful content reduction in exhaust gases is based on the implementation of the modern and accurate automatic combustion control systems that perform real-time measurements of the combusting torch parameters.

In order to measure the torch combustion parameters, contact [1] or non-contact (e.g. PIV systems [2]) sensors are traditionally used. The contact sensors are usually mounted inside the 
torch that results in transformation of the torch heat and mass transfer mode. Also, the problem of positioning a sensor that works under high thermal stress conditions arises. Therefore, the contact torch measuring methods are inappropriate for industrial application, and they are usually implemented only in laboratory studies of the flames. The non-contact methods usually employ optical cameras; they provide information about the field of measuring parameter, instead of the point in contact methods. However, in present these technologies are also implemented only in laboratory studies due to the problem of the obtained data processing, in particular quantitative parameters extraction from the initial data.

A matter of major interest in non-contact torch investigation is optical investigation in infrared (IR) band. This approach is fulfilled by using IR cameras, i.e. thermal imagers, providing registration of the sequences of instant IR images of the torch. The instant image of the torch is projected onto two-dimensional system of photo sensors. Then the sensor output values (traditionally measured in Volts) are calculated to the temperature values of the investigated object surface, and the temperature field of this surface restored as a two-dimensional image [3].

It should be noted, that it is impossible to calculate a real temperature field of the burning torch using IR imaging, because the emissive coefficients of the torch surface are unknown. In this case it is assumed that the torch radiance is measured in the relative units - relative Celsius degrees. Then the recorded temperature field or its transformation into another feature space is visualized as an image representing the color map. The examples of typical experimental data visualization are shown on fig. 1.


Figure. 1. The visualization of instant IR images of the gaseous fuel torch(temperature field $T_{i, j}$ ) in various feature spaces: a) the standard deviation of the temperature ${ }_{T_{i, j}}$; ) the field of temperature pulsations frequency $f_{i, j}, 8$ ) the field of temperature pulsations phase; 2 ) the field of two-dimensional Wavelet transform coefficients of the initial IR sequence [5]

Fig. 1 shows that the shapes of the torch are different depending on the feature space. Also, in the space of pulsations phase as well as in the space of wavelet coefficients the torch is represented as a composition of the close to each other values segments. This result, according to the authors of original investigation [5] demonstrates the turbulent structure of the flame. However, there are no such structures on 1a, 16 images. Hence, the torch shape and the combustion pa- 
rameters depend on the feature space, and transfer between feature spaces does not simplify the quantitative parameters extraction.

Therefore, the study of the torch IR data analysis approaches in order to choose the quantitative parameters extraction methods is a problem of great interest due to application in modern automated combustion control systems.

The paper substantiates the choice of the torch IR data processing methods that allow to extract the quantitative parameters of the gaseous fuel torch combustion.

\section{The experiment study and obtained data analysis}

To perform the study the experimental complex was constructed. This complex makes possible to obtain instant IR images of the gaseous fuel torch at given frequency (IR video record). This complex has been described in details in [6], and the experimental complex scheme is shown at fig. 2.

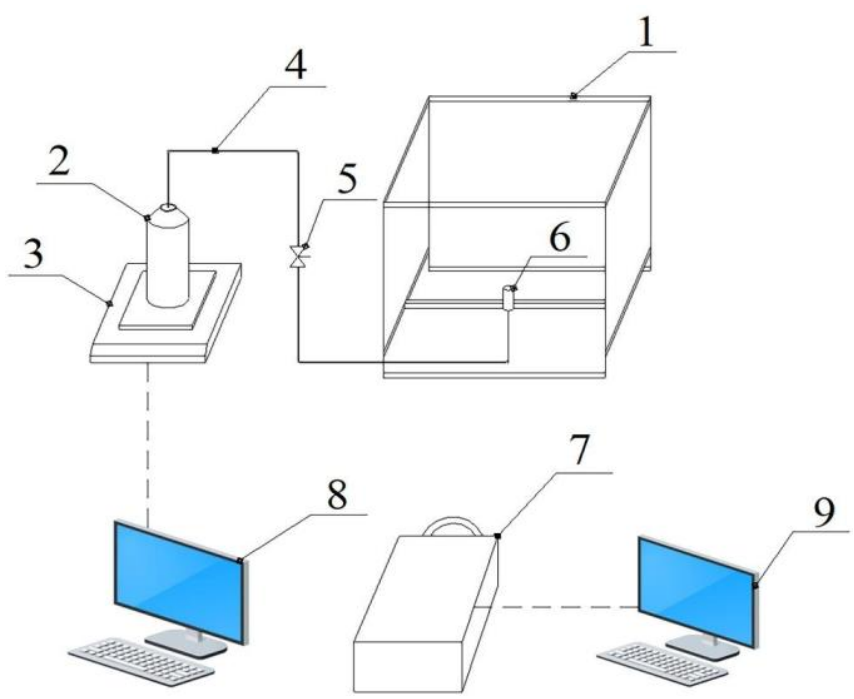

Figure 2. Scheme of the experimental complex: 1 - the torch area frame, 2 - gas fuel container, 3 - the scales, 4 - connection tubes, 5 - ball valve, 6 - the burner, 7 - thermal imager, 8,9-operating personal computers

In that experimental complex the FLIR7700M thermal imager is used, that obtain IR radiation in the $1.5-5.1 \mu \mathrm{m}$ wavelength. This thermal imager provides recording of IR images sequences of the torch in resolution of $320 \times 256$ pixels with frequency of $412 \mathrm{~Hz}$, the record duration is 10 seconds.

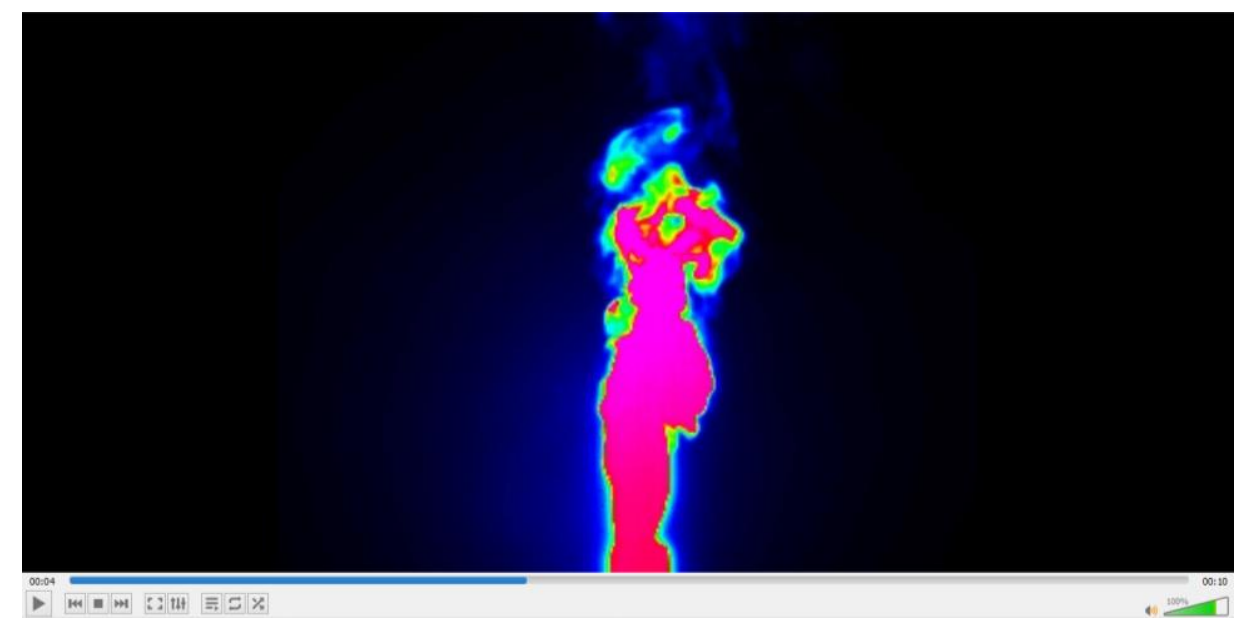

Figure 3. Visualization of the obtained initial IR images sequence $T_{i, j, k}$ of the burning torch The results of the burning torch IR images visualization using "Rainbow" color map are presented on fig. 4 (844-th, 846-th, 848-th and 850-th frames of the initial IR sequence). 


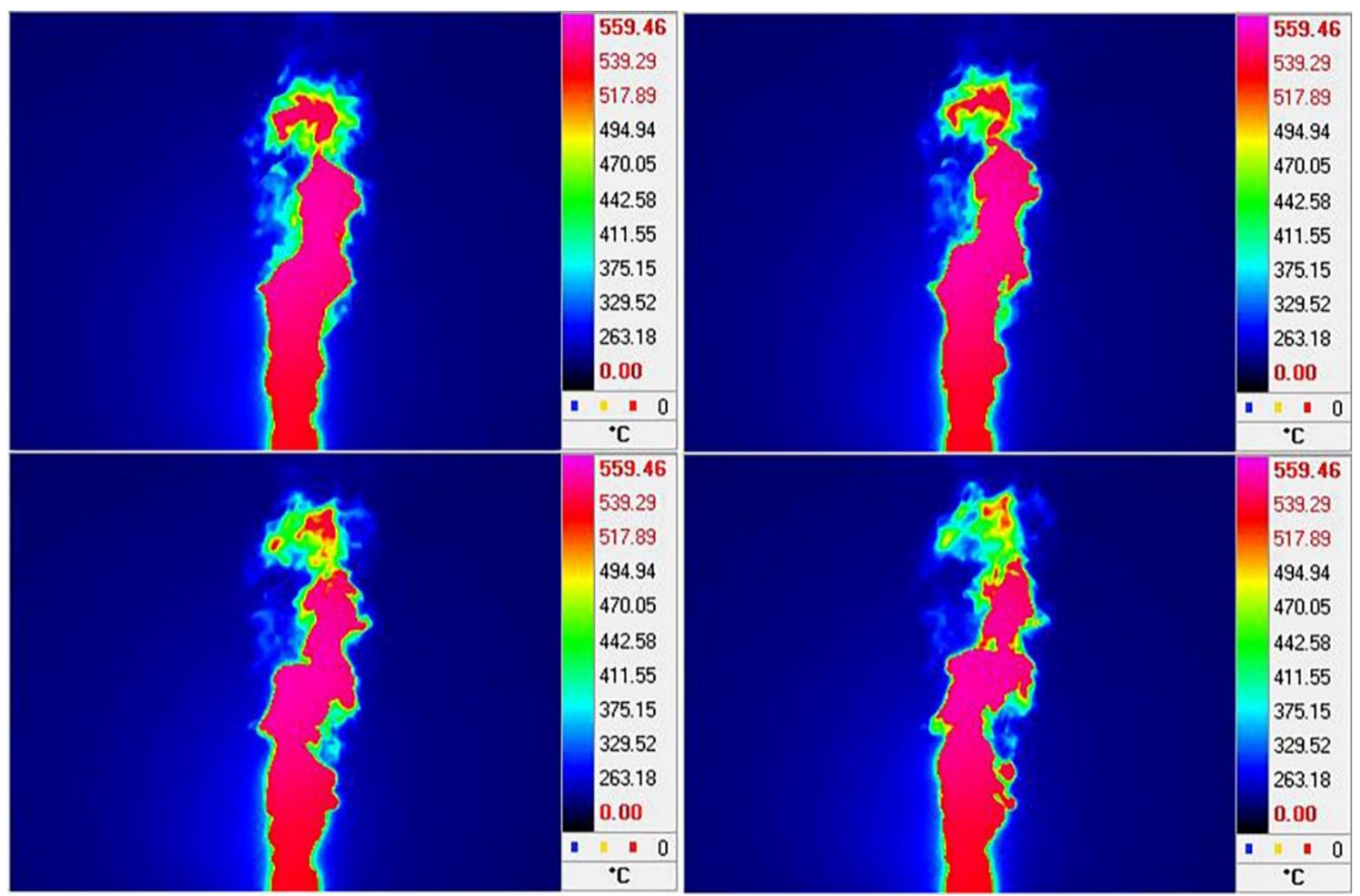

Figure 4. The visualization (using "Rainbow" color map) of the IR images of the burning torch under continuous mode of the gaseous fuel supply mode: the interval between adjacent frames is $\Delta \tau=0,00485$ seconds

Mathematically, the initial IR sequences are three-dimensional arrays $T_{i, j, k}$ with size of $320 \times 256 \times 4120(i, j-$ pixels quantity in the $X o Y$ plane, $k$ - frame number). Typical pixels distribution on the the ranges of the relative temperature values is shown on fig. 5 .
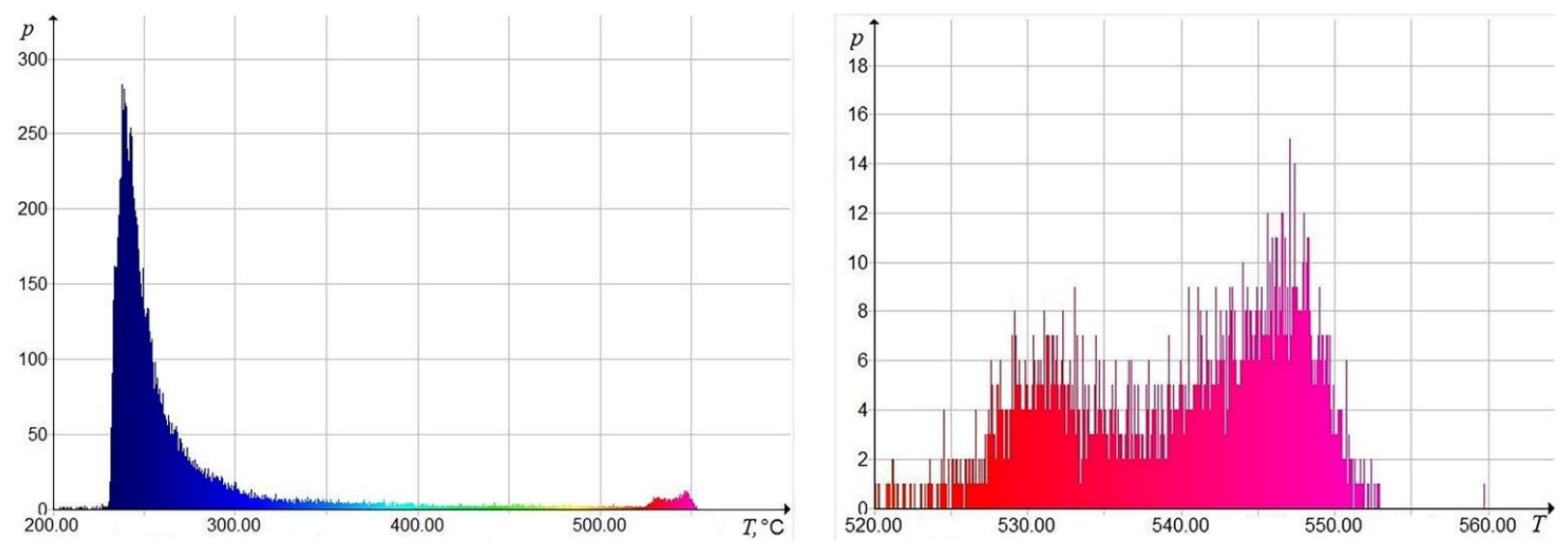

Figure 5. Histogram (left) and its fragment (right) of the series $\tilde{T}_{k}$

Fig. 5. shows that on the initial IR image of the burning torch three groups of pixels can be distinguished, with values in the ranges of $[230,320],[320,520]$, [520,560] relative Celsius degrees consequently.

The analysis of the burning torches IR images showed that:

- Pixels with values in the range of [230,320] relative Celsius degrees represent background;

- Pixels with values in the range of [320,520] relative Celsius degrees are located at the torch borders; 
- Pixels with values in the range of [520,560] relative Celsius degrees are located in the area of the torch.

Also, fig. 5 shows that pixels distribution density $\tilde{p}(T)$ of the series $\tilde{T}_{k}$ (where $\tilde{T}_{k} \in[520,560]$ relative Celsius degrees, $k$ - serial number of the pixel) can be approximated by the line with two local maximums $\left(\tilde{T}_{\max }^{\langle 1\rangle}, \tilde{T}_{\max }^{\langle 2\rangle}\right)$ and local minimum $\left(T_{\min }\right)$ located between them. This feature of the $\tilde{p}(T)$ distribution density makes possible to consider the series $\tilde{T}_{k}$ as a random series with bimodal distribution law.

To compute the approximations of the $\tilde{T}_{k}$ distribution density, in accordance with [7] the nonparametric method - kernel density estimation (KDE) was applied. This method, also known as Rosenblatt - Parzen approximation, is implemented in the MATLAB program library ES\&RP [8].

Approximation of the $\tilde{T}_{k}$ series distribution density was computing in accordance with the following algorithm.

1) Selection of the current frame $T_{k=\text { const }, i, j}$ from the initial IR sequence.

2) Composition of the series $T_{k=\text { const }, l}$ by deployment along the row of the selected frame $T_{k=\text { const }, i, j}$.

3) Composition of the series $\tilde{T}_{k=\text { const }, \tilde{l}}$ by deleting values less than 520 relative Celsius degrees.

4) Computing of the $\tilde{T}_{k=\text { const }, l}$ series distribution density using KDE method.

In the $\mathrm{KDE}$ method restoring (approximation) of the distribution function of the random sequence $x_{i}$, where $i=\overline{1, N s}$, is found as:

$$
F(\xi)=\frac{1}{N s} \sum_{i=1}^{N s} K\left(\frac{\xi-x_{i}}{h}\right),
$$

where $K(t)$ is a kernel function that agrees with following features:

_ $K(t)$ _ monotonically decreasing function, with range of values in $[0,1]$ interval;

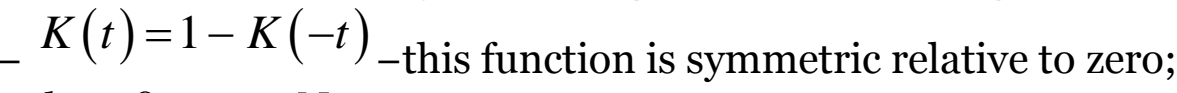

- $h \rightarrow 0$ when $N s \rightarrow \infty$;

$\mathrm{h}$ - scattering factor, that defines smoothness of the evaluation of distribution function. (If the scattering factor is non-optimal, the Rosenblatt - Parzen approximation will deviate from the true distribution function).

Respectively, the distribution density of the random series $x_{i}, i=\overline{1, N s}$, is calculated as:

Where

$$
p(\xi)=\frac{1}{N s \cdot h} \sum_{i=1}^{N s} k\left(\frac{\xi-x_{i}}{h}\right),
$$

$$
k(\xi)=\frac{d}{d \xi} K(\xi) .
$$


Since in the KDE method a range of kernel functions $k(t)$ is commonly used: uniform, triangular, biweight, triweight, Epanechnikov, normal, and others, there are two tasks to be solved in the KDE procedure:

1) choice of the optimal value for the smoothing parameter for the current kernel function.

2) choice of the kernel function which provides the best accuracy of the distribution density approximation.

In [7] it was suggested to choose optimal value of the smoothing parameter $h^{*}$ by solving the equation:

$$
h^{*}=\arg \max \left(\frac{1}{N s} \sum_{i=1}^{N s} \ln \left[\frac{1}{(N s-1) \cdot h} \sum_{i \neq j}^{N s} k\left(\frac{x_{i}-x_{j}}{h}\right)\right]\right),
$$

and then choose the function that has the maximum value

$$
\varphi(h)=\frac{1}{N s} \sum_{i=1}^{N s} \ln \left(\frac{1}{(N s-1) \cdot h} \sum_{i \neq j}^{N s} k\left(\frac{x_{i}-x_{j}}{h}\right)\right)
$$

at the $h=h^{*}$ condition.

The experience of ES\&RP library implementation showed that calculation of the $\tilde{T}_{k}$ series density distribution approximation even for only one IR frame requires significant time excess (up to 417 seconds and more). Therefore, preliminarily 120 frames of the initial IR sequence were selected and density distribution approximations of the $\tilde{T}_{k=\text { const }, \tilde{l}}$ series using KDE method and each of the common kernel functions was calculated. Then the values of the function $\varphi_{m}\left(h^{*}\right)$ were analyzed ( $m$ - is a number of the index of the current kernel function), and it was figured out that on all 120 frames the maximum value of the function $\varphi(h)$ was reached when the normal distribution was used as a kernel function. This result allowed to use only one (the best for our case) kernel function that reduced the total processing time from 417 to 232 seconds.

An example of calculated KDE approximation of the $\tilde{T}_{k=\text { const }, \tilde{l}}$ series density distribution, using normal distribution as a kernel function is shown on fig. 6.

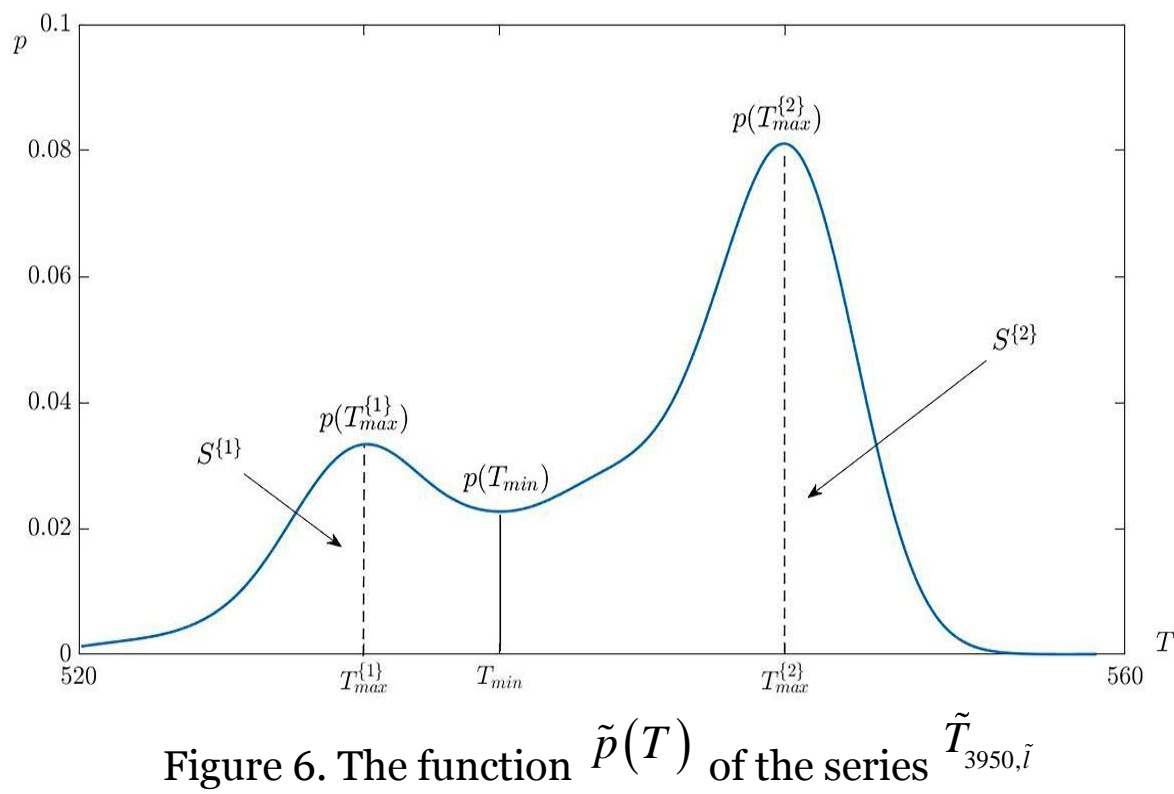


Fig. 6 shows that the distribution can be described by the abscissa values of three local extreme points $T_{\max }^{\{1\}}, T_{\max }^{\{2\}}, T_{\min }$ and related values of the function at these points: $\tilde{p}_{\max }^{\{1\}}=\tilde{p}\left(T_{\max }^{\{1\}}\right)$, $\tilde{p}_{\max k}^{\{2\}}=\tilde{p}_{k}\left(T_{\max k}^{\{2\}}\right), \tilde{p}_{\min }=\tilde{p}\left(T_{\min }\right)$, and the value $S^{\{1\}}$ :

$$
S^{\{1\}}=\int_{520}^{T_{\min }} \tilde{p}(T) d T,
$$

and $S^{\{2\}}$ :

$$
S^{\{2\}}=\int_{T_{\min }}^{560} \tilde{p}(T) d T .
$$

Knowing the values of (6), (7), we can also calculate the quantity of the pixels $N^{\{1\}}, N^{\{2\}}$, with values in the ranges of $\left[520 ; T_{\min }\right]$ and $\left[T_{\min } ; 560\right]$ relative Celsius degrees, respectively:

$$
\begin{aligned}
& N^{\{1\}}=S^{\{1\}} \cdot N^{\{0\}} \\
& N^{\{2\}}=S^{\{2\}} \cdot N^{\{0\}}
\end{aligned}
$$

where $N^{\{0\}}$ is the quantity of the pixels with values in the ranges of [520;560] relative Celsius degrees.

Then we performed an analysis of the pixels location with values in the ranges of $\left[520 ; T_{\min }\right]$ and $\left[T_{\min } ; 560\right]$ relative Celsius degrees at the initial IR frame. The analysis of the result demonstrated that these pixels are located in two zones respectively, and these zones may be approximated by the curves with no intersections and self-intersections (fig. 7).


Figure 7. The visualization of the pixels zones, with values in the ranges of ${ }^{\left[520 ; T_{\min }\right]}$ (left) and $\left[T_{\min } ; 560\right]$ (right) relative Celsius degrees

The comparison of the obtained result with the known thermal-fluid dynamics torch combustion models showed that allocated zones in the torch IR image correspond with the physical concepts of the torch structure [9].

Using the selected quantitative indicators that characterize the instantaneous state of the torch, the researchers can describe the dynamics of torch fuel combustion. To do so the time sequences (TS) $T_{\max k}^{\{1\}}, T_{\max k}^{\{2\}}, T_{\min k}, p_{\max k}^{\{1\}}, p_{\max k}^{\{2\}}, p_{\min k}, N_{k}^{\{0\}}, N_{k}^{\{1\}}, N_{k}^{\{2\}}$, should be compared by computing the values of the related quantitative indicators for each frame of the IR record of the 
torch. To describe the torch fuel combustion in terms of their statistical and frequency-temporal characteristics one should make a transition from the $3 \mathrm{D}$ matrix $T_{i, j, k}$ to these time sequences.

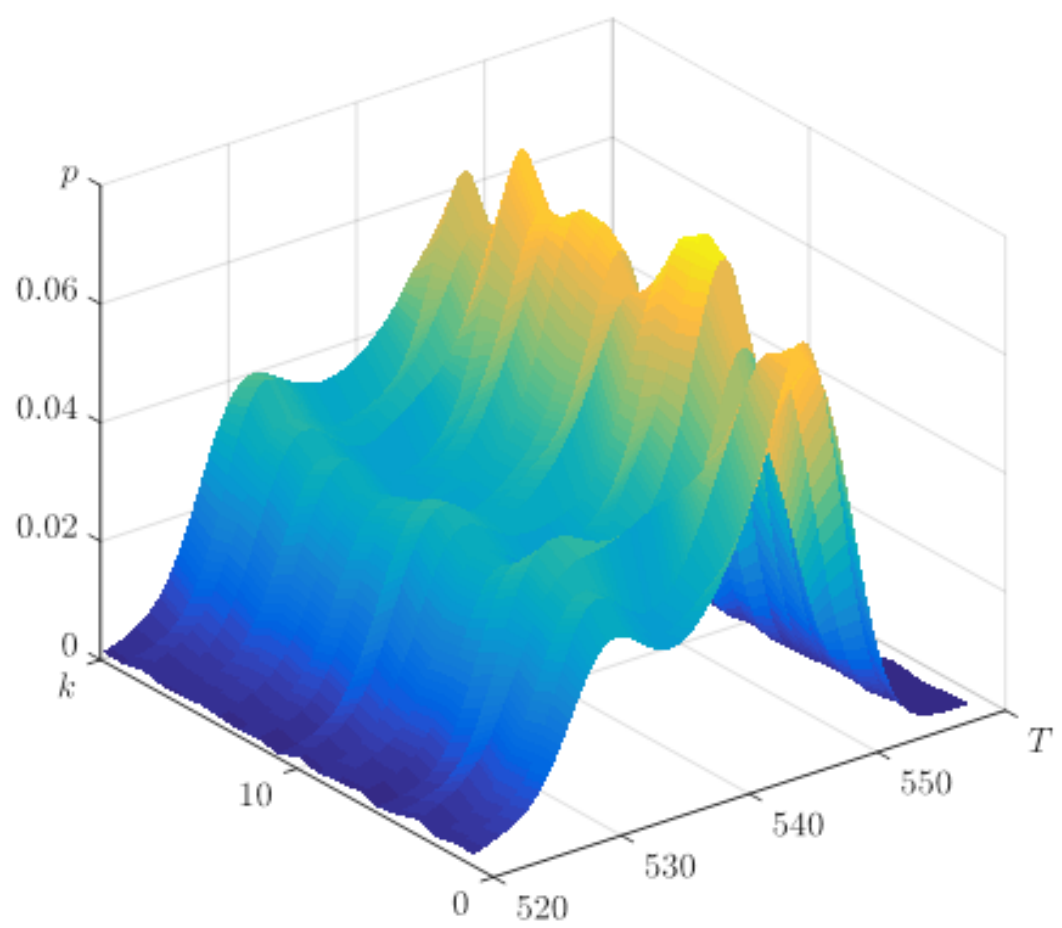

Figure 8. Graphical representation of the surface built through approximations of distribution density sequences $\tilde{T}_{k=\text { const }, \tilde{I}}$ with the time interval between adjacent frames of $0.0121 \mathrm{sec}$.

\section{Verification of stationarity of the selected quantitative in- dicators}

Based on the analysis of the surface generated by approximations of the time sequences distribution density $\tilde{T}_{k, \tilde{l}} \quad k=\overline{1,4120}$, (Fig. 8) we draw a conclusion that there are differences between functions $\tilde{p}_{k}(T)$ that manifest themselves by different values of $T_{\max }^{\{1\}}, T_{\max }^{\{2\}}, T_{\min }, p_{\max }^{\{1\}}, p_{\max }^{\{2\}}$, $p_{\min }$ in different frames. This conclusion is supported by visual analysis of dependencies of the selected quantitative indicators from time in some random sequences $T_{\max k}^{\{1\}}, T_{\max k}^{\{2\}}, T_{\min k}$, $p_{\max k}^{\{1\}}, p_{\max k}^{\{2\}}, p_{\min k}, N_{k}^{\{0\}}, N_{k}^{\{1\}}, N_{k}^{\{2\}}$. The detection of this phenomenon necessitated verification of their stationarity.

The study of stationarity of time sequences $T_{\max k}^{\{1\}}, T_{\max k}^{\{2\}}, T_{\min k}, p_{\max k}^{\{1\}}, p_{\max k}^{\{2\}}, p_{\min k}, N_{k}^{\{0\}}$, $N_{k}^{\{1\}}, N_{k}^{\{2\}}$ employed the procedure described on the example of TS BP $T_{\max k}^{\{1\}}, T_{\max k}^{\{2\}}, T_{\min k}$ :

1) Three subsequences $T_{i, j, k}^{\text {начало }}, T_{i, j, k}^{\text {середина }}{ }_{\text {и }} T_{i, j, k}^{\text {кони }}$ were made, each 40 frames long. These subsequences were extracted from the beginning, middle and end segments of the original IR record $T_{i, j, k}$, which was 10 second long. Samples were taken once each 5 frames, which equals to the time interval of $0.0121 \mathrm{sec}$. 
2) Each frame of the subsequences $T_{i, j, k}^{\text {начало }}, T_{i, j, k}^{\text {середина }}, T_{i, j, k}^{\text {конеи }}$ was subjected to horizontal scanning to make sequences $T_{k=\text { const }, l}^{\{\text {начало }\}}, T_{k=\text { const }, l}^{\{\text {середин }\}}, T_{k=\text { const }, l}^{\{\text {коне }\}}$ with their subsequent transformation to sequences $\tilde{T}_{k=\text { сопst }, \tilde{l}}^{\{\text {начал }\}}, \tilde{T}_{k=\text { const }, \tilde{l}}^{\{\text {середи }\}}, \tilde{T}_{k=\text { const }, \tilde{l}}^{\{\text {конеи }\}}$ as per the described procedure.

3) Determination of Rosenblatt - Parzen approximations $\tilde{p}(T)$ for each sequence $\tilde{T}_{k=\text { сопst }, \tilde{l}}^{\text {Һачало }}$, $\tilde{T}_{k=\text { const }, \tilde{l}}^{\{\text {середин }\}}, \tilde{T}_{k=\text { const }, \tilde{l}}^{\{\text {конеи }\}}$ obtained at phases 1 and 2.

4) Determination of x-coordinate of the local extrema $T_{\max k}^{\{1\}}, T_{\max k}^{\{2\}}, T_{\min k}$ of Rosenblatt Parzen approximations $\tilde{p}(T)$ for each of the sequences $\tilde{T}_{k=\text { const }, l}^{\{\text {началo }\}}, \tilde{T}_{k=\text { const }, l}^{\{\text {середин }\}}, \tilde{T}_{k=\text { const }, l}^{\{\text {, obец }\}}$ tained at the phase 3 of this procedure.

Fig. 9 shows time sequences $T_{\max k}^{\{1\}}, T_{\max k}^{\{2\}}, T_{\min k}$ computed for the beginning, middle and the end segments of the burning torch IR imaging. Components of these sequences were obtained through the procedure described above.
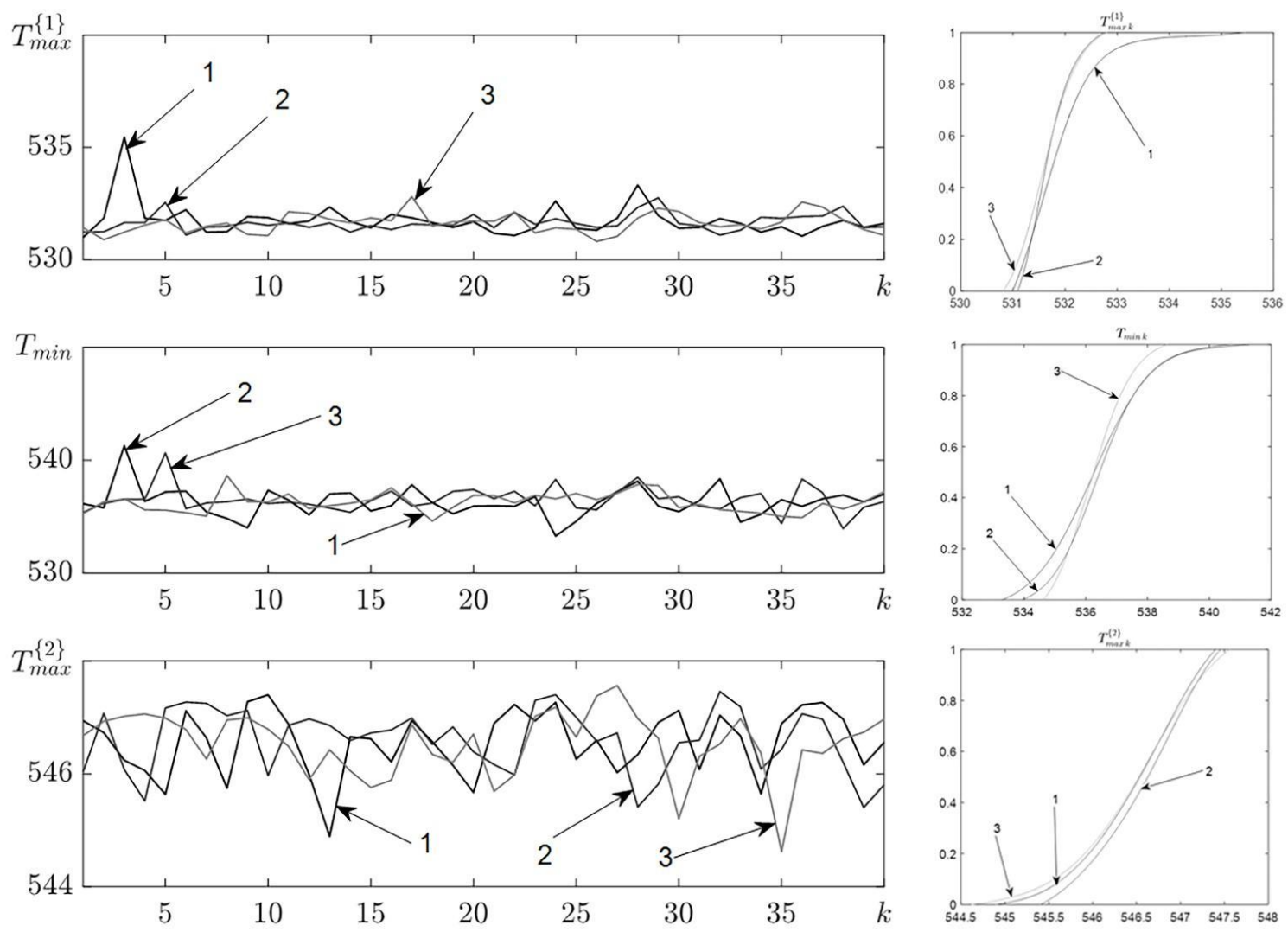

Figure 9. BP $T_{\max k}^{\{1\}}, T_{\min k}, T_{\max k}^{\{2\}}$ (left) and their distribution functions (right) at the selected segments of the IR thermal imager record: 1 - beginning segment, 2 - middle segment, 3 - end segment.

Then the approximation quantiles of TS distribution functions $T_{\max k}^{\{1\}}, T_{\max k}^{\{2\}}, T_{\min k \text { и }} p_{\max k}^{\{1\}}$, $p_{\min k}, p_{\max k}^{\{2\}}, N_{k}^{\{0\}}, N_{k}^{\{1\}}, N_{k}^{\{2\}}$ were calculated through the Rosenblatt - Parzen method. The above functions are those for which the confidence levels are 0.05, 0.5 and 0.95 as shown in Table 1 . 
Table 1. Quantiles of Rosenblatt - Parzen approximations of time sequences distribution functions $T_{\max k}^{\{1\}}, T_{\max k}^{\{2\}}, T_{\min k \text { и }} p_{\max k}^{\{1\}}, p_{\min k}, p_{\max k}^{\{2\}}, N_{k}^{\{0\}}, N_{k}^{\{1\}}, N_{k}^{\{2\}}$

\begin{tabular}{|c|c|c|c|c|c|c|c|}
\hline \multirow{2}{*}{ Parameter } & \multirow{2}{*}{$\begin{array}{l}\text { Segment of the } \\
\text { IR thermal im- } \\
\text { ager }\end{array}$} & \multicolumn{3}{|c|}{ Confidence level } & \multicolumn{3}{|c|}{$\begin{array}{l}\text { Relative deviation of quan- } \\
\text { tiles, } \%\end{array}$} \\
\hline & & 0.05 & 0.5 & 0.95 & 0.05 & 0.5 & 0.95 \\
\hline \multirow{3}{*}{$T_{\max }^{\{1\}}$} & Beginning & 531.09 & 531.79 & 533.13 & \multirow{3}{*}{0.02} & \multirow{3}{*}{0.02} & \multirow{3}{*}{0.07} \\
\hline & Middle & 531.17 & 531.65 & 532.44 & & & \\
\hline & End & 530.95 & 531.62 & 532.47 & & & \\
\hline \multirow{3}{*}{$T_{\min }$} & Beginning & 534.00 & 536.27 & 538.84 & \multirow{3}{*}{0.09} & \multirow{3}{*}{0.02} & \multirow{3}{*}{0.09} \\
\hline & Middle & 534.60 & 536.45 & 538.76 & & & \\
\hline & End & 534.90 & 536.25 & 537.94 & & & \\
\hline \multirow{3}{*}{$T_{\max }^{\{2\}}$} & Beginning & \begin{tabular}{|l|l|}
545.42 \\
\end{tabular} & 546.54 & 547.28 & \multirow{3}{*}{0.03} & \multirow{3}{*}{0.01} & \multirow{3}{*}{0.01} \\
\hline & Middle & 545.63 & 546.63 & 547.34 & & & \\
\hline & End & 545.27 & 546.52 & 547.39 & & & \\
\hline \multirow{3}{*}{$p_{\max }^{\{1\}}$} & Beginning & 0.0275 & 0.0344 & 0.0424 & \multirow{3}{*}{6.59} & \multirow{3}{*}{2.57} & \multirow{3}{*}{4.21} \\
\hline & Middle & 0.0313 & 0.0358 & 0.0405 & & & \\
\hline & End & 0.0289 & 0.0341 & 0.0390 & & & \\
\hline \multirow{3}{*}{$p_{\min }$} & Beginning & 0.0234 & 0.0294 & 0.0364 & \multirow{3}{*}{$3 \cdot 35$} & \multirow{3}{*}{1.05} & \multirow{3}{*}{1.94} \\
\hline & Middle & 0.0232 & 0.0299 & 0.0374 & & & \\
\hline & End & 0.0247 & 0.0300 & 0.0360 & & & \\
\hline \multirow{3}{*}{$p_{\max }^{\{2\}}$} & Beginning & 0.0495 & 0.0652 & 0.0805 & \multirow{3}{*}{4.54} & \multirow{3}{*}{1.03} & \multirow{3}{*}{3.21} \\
\hline & Middle & 0.0530 & 0.0643 & 0.0765 & & & \\
\hline & End & 0.0540 & 0.0655 & 0.0759 & & & \\
\hline \multirow{3}{*}{$N_{k}^{\{1\}}$} & Beginning & 1329 & 1782 & 2450 & \multirow{3}{*}{7.05} & \multirow{3}{*}{1.54} & \\
\hline & Middle & 1312 & 1729 & 2283 & & & 7.11 \\
\hline & End & 1488 & 1747 & 2125 & & & \\
\hline & Beginning & 2777 & 3903 & 4928 & & & \\
\hline$N_{k}^{\{2\}}$ & \begin{tabular}{|l|} 
Middle \\
\end{tabular} & 2859 & 3578 & 4375 & 6.90 & 4.73 & 6.21 \\
\hline & End & 3161 & 3871 & 4815 & & & \\
\hline & Beginning & 4985 & 5667 & 6438 & & & \\
\hline$N_{k}^{\{0\}}$ & Middle & 4683 & 5323 & 6060 & 3.18 & 3.29 & 3.35 \\
\hline & End & 4891 & 5597 & 6411 & & & \\
\hline
\end{tabular}

The table 1 demonstrates that

1) The quantiles of approximations of distribution functions for time sequences $T_{\max k}^{\{1\}}, T_{\max k}^{\{2\}}$, $T_{\min k}, p_{\min k}$ are close to each other. Therefore, statistically the time sequences under study are partial samples extracted from the corresponding sampled population.

2) The quantiles of approximations of distribution function for the time sequence $T_{\min k}$ at confidence levels $0.05,0.5,0.95$ differ for no more than $1.8 \%$. Therefore, the quantitative parameter $T_{\min }$ can be assumed a constant equal to 536.3. This result allows to calculate quantitative indicators of the combustion process $N^{\{0\}}, N^{\{1\}}, N^{\{2\}}$ and make corresponding time sequences without running Rosenblatt - Parzen approximation of sequences $\tilde{T}_{k, \tilde{l}}$. 
Due to relatively large differences between quantiles of distribution functions at the confidence levels of 0.05, 0.5, 0.95 for time sequences $N_{k}^{\{0\}}, N_{k}^{\{1\}}, N_{k}^{\{2\}}$, similar values of time sequences $N_{k}^{\{0,1\}}=N_{k}^{\{0\}}, \quad N_{k}^{\{0,2\}}=N_{k+2060}^{\{0\}}, \quad N_{k}^{\{1,1\}}=N_{k}^{\{1\}}, \quad N_{k}^{\{1,2\}}=N_{k+2060}^{\{1\}}, \quad N_{k}^{\{2,1\}}=N_{k}^{\{2\}}, \quad N_{k}^{\{2,2\}}=N_{k+2060}^{\{2\}}$, $k=\overline{1,2060}$ were found. Comparative analysis of those shows that the maximum deviations between quintiles of distribution functions of time sequences $N_{k}^{\{0,1\}}=N_{k}^{\{0\}}, N_{k}^{\{0,2\}}=N_{k+2060}^{\{0\}}$, $N_{k}^{\{1,1\}}=N_{k}^{\{1\}}, N_{k}^{\{1,2\}}=N_{k+2060}^{\{1\}}, N_{k}^{\{2,1\}}=N_{k}^{\{2\}}, N_{k}^{\{2,2\}}=N_{k+2060}^{\{2\}}, k=\overline{1,2060}$ at confidence levels of $0.05,0.5,0.95$ is 2.87\%. This confirms stationarity of time sequences $N_{k}^{\{0\}}, N_{k}^{\{1\}}, N_{k}^{\{2\}}$. In addition, the assumption of stationarity of time sequences $T_{\max k}^{\{1\}}, T_{\max k}^{\{2\}}, T_{\min k}, p_{\max k}^{\{1\}}, p_{\max k}^{\{2\}}$, $p_{\min k}, N_{k}^{\{0\}}, N_{k}^{\{1\}}, N_{k}^{\{2\}}$ was confirmed through the use or Kwiatkowski-Phillips-Schmidt-Shin test [10]. To run the test the authors used the function kpss.m that is included in the MATLAB toolkit Econometrics Toolbox [11].

\section{Analysis of the selected quantitative indicators of the combustion process under pulse mode fuel supply conditions}

We tested the hypothesis that the selected set of indicators can be used for description of combustion process where fuel is supplied in pulsating mode. This mode is of practical interest because it helps reduce content of environmentally noxious nitrogen oxides that can be found in gaseous fuel combustion products [12].

In these experiments we used an upgraded test unit with a solenoid valve installed in the fuel supply line. The valve controlled the fuel flow rate by fast (as compared with durations of its open/closed states) opening and closing at the rate from o to 10 Hertz (pulsating fuel supply mode). The average fuel supply flow rate was maintained the same as in the continuous fuel supply mode (0.07 g/sec).

Analysis of the obtained IR images demonstrated that where the valve opening/closing rate was $1 \mathrm{~Hz}$, the gas-air mixture burning process was unstable, causing the torch to shut down. Due to that, we further studied only those IR image sequences $T_{i, j, k}$ where the valve opening/closing rate was in the range of 2 to $10 \mathrm{~Hz}$. Figure 10 shows an example of IR imaging record for the pulsating fuel supply mode.

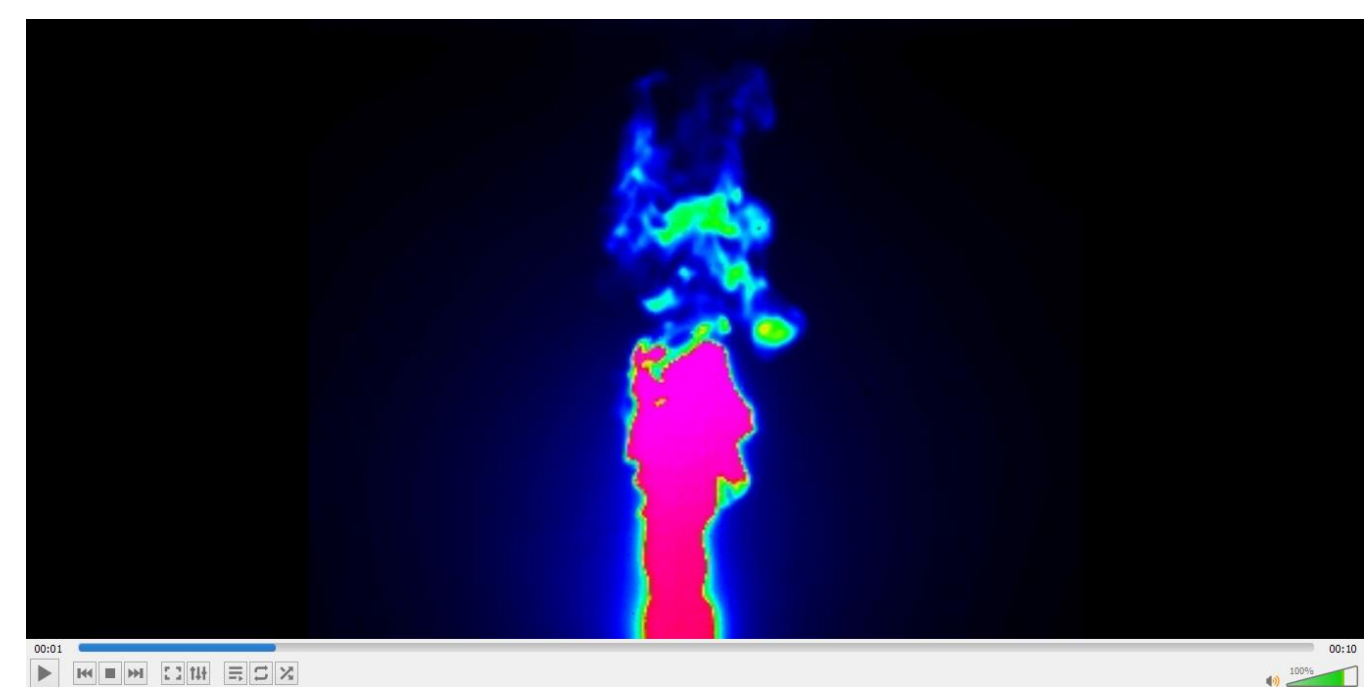

Figure 10. Visualization of the IR heat imager sequence $T_{i, j, k}$ for a burning torch where the fuel is supplied to the burner at the pulsation rate of $2 \mathrm{~Hz}$ 
Figure 11 shows visualization of IR images of the burning torch operating in the pulsating mode. This visualization resides in the relative Celsius degrees space using the color scale «Rainbow» at the 500th, 502th, 504th и 506th frames of the IR image recording, fragments of which are shown on Fig. 10.
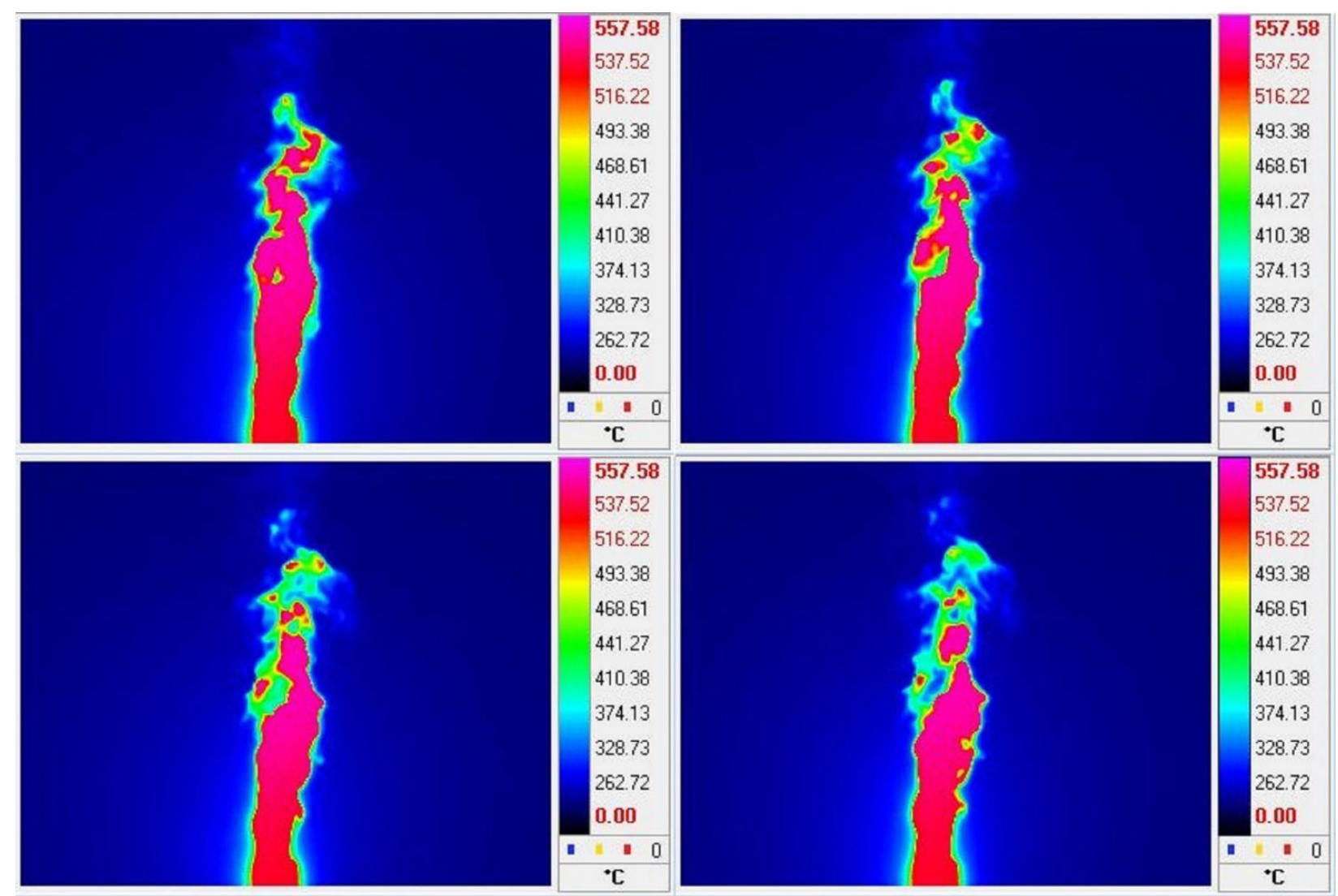

Figure 11. Visualization of IR images of the burning torch operating in pulsating mode at the rate of $2 \mathrm{~Hz}$ using the color scale Rainbow. Time interval between adjacent frames is

$$
\Delta \tau=0,00485 \text { sec. }
$$

Fig. 10 and 11 suggest that the raw data obtained at IR imaging of the torch operating in pulsating fuel supply mode does not differ visually from data obrained in continuous fuel supply. Therefore, following the procedure descibed in section 2 the authors performed analysis of the raw data - sequences of IR images containing images of burning torch. Fig. 12 depicts a typical example of surface generated by Rosenblatt - Parzen approximations of density distributions for sequences $\tilde{T}_{k=\text { const }, \tilde{l}}$, computed for a 0.242 seconds-long interval taken from the image sequence $T_{i, j, k}$ for pulsating mode of fuel supply with the pulsating rate of $2 \mathrm{~Hz}$. 




Figure. 12. Image of the surface generated through approximations of Rosenblatt - Parzen distribution density for sequences $\tilde{T}_{k=\text { const, }, \tilde{I}}$ (time interval between adjacent frames is 0.0121 seconds). The IR image sequences were obtained when operating the torch in pulsating fuel supply mode with the pulsation rate of $2 \mathrm{~Hz}$.

As follows from Fig. 12, this surface is shaped similarly to that generated for the continuous fuel supply option (Fig. 8). This means that for the pulsating fuel supply mode the approximations of sequences $\tilde{T}_{k=\text { const }, \tilde{l}}$ are bimodal random sequences with a constrained scattering region. Distribution densities of these sequences are characterized by the pre-selected set of parameters: $T_{\max }^{\{1\}}, T_{\max }^{\{2\}}, T_{\min }, p_{\max }^{\{1\}}, p_{\min }, p_{\max }^{\{2\}}, N^{\{0\}}, N^{\{1\}}, N^{\{2\}}$. This conclusion is also confirmed by the fact that for the pulsating mode, as well as for the continuous fuel supply mode, it is advisable to use normal kernel in computations of Rosenblatt - Parzen approximations of the distribution density for sequences $\tilde{T}_{k=\text { const }, \tilde{l}}$. It should be noted that the analysis of time sequences $T_{\max k}^{\{1\}}$, $T_{\max k}^{\{2\}}, T_{\min k}, p_{\max k}^{\{1\}}, p_{\max k}^{\{2\}}, p_{\min k}, N_{k}^{\{0\}}, N_{k}^{\{1\}}, N_{k}^{\{2\}}$, performed following the procedure described in section 3, confirmed their stationarity. In addition, the stationarity of time sequences in the range of the control valve opening/closing rates of $2 \ldots 10 \mathrm{~Hz}$ was confirmed by the running the Kwiatkowski-Phillips-Schmidt-Shin test.

The results shown here demonstrate that the suggested quantitative characteristics are universal parameters of the burning torch regardless of the studied gaseous fuel supply modes. Availability of these parameters makes it possible to develop an automatic burning process control system based on IR thermal imagers.

\section{Conclusion}

The analysis of IR images of burning torch with continuous supply of gaseous fuel has demonstrated that the combustion process is characterized by the following set of qualitative indicators evaluated through Rosenblatt - Parzen approximations of the pixel distributions of torch images at the given frame on the relative temperature: 
$T_{\max }^{\{1\}}-\mathrm{x}$-coordinate of the first maximum, $T_{\max }^{\{2\}}-\mathrm{x}$-coordinate of the second maximum, $T_{\min }$ the value of the minimum, $p_{\max }^{\{1\}}-\mathrm{y}$-coordinate of the first maximum, $p_{\max }^{\{2\}}-\mathrm{y}$-coordinate of the second maximum, $p_{\min }$ - y-coordinate of the minimum, ${ }^{\{0\}}$ - number of pixels with values in the range of $[520,560]$ relative ${ }^{\circ} C, N^{\{1\}}$ - number of pixels with values in the range of $\left[520, T_{\min }\right]$ relative ${ }^{\circ} \mathrm{C}, N_{k}^{\{2\}}$ - number of pixels with values in the range of $\left[T_{\min }, 560\right]$ relative ${ }^{\circ} \mathrm{C}$.

Time sequences $N_{k}^{\{0\}}, N_{k}^{\{1\}}, N_{k}^{\{2\}}, T_{\max k}^{\{1\}}, T_{\max k}^{\{2\}}, T_{\min k}, p_{\max k}^{\{1\}}, p_{\max k}^{\{2\}}, p_{\min k}$, composed from the values of the selected quantitative indicators of the combustion process, which were calculated for each frame of the image sequences, are stationary random sequences.

The finding that $T_{\min }$ with the relative error of less than $2.8 \%$ is a constant equal to 536.3 relative ${ }^{\circ} \mathrm{C}$ opens the opportunity to reduce the scope of real-time mathematical operations to be performed by the combustion control system. The time sequences $N_{k}^{\{0\}}, N_{k}^{\{1\}}, N_{k}^{\{2\}}$ can be found without Rosenblatt - Parzen approximation of distribution of pixels in IR-images of the torch, which is rather resource-consuming procedure. Instead, this allows to directly cound the number of pixels that represent temperatures in the ranges $\left[520, T_{\min }\right],\left[T_{\min }, 560\right],[520,560]$ of relatives ${ }^{\circ} \mathrm{C}$.

\section{References}

1) Fristrom R. M. Struktura plameni / R. M. Fristrom, A. A. Vestenberg. - Moscow : Metallurgiya, 1969. - 364 p. [in Russian]

2) Primenenie sovremennyh opticheskih metodov dlya diagnostiki i prostranstvennoj struktury turbulentnyh plamyon / V. M. Dulin, D. M. Markovich, M. P. Tokarev, L. M. CHikishev // Avtometriya. - 2012. - V. 48, № 3. - P. 22-32. [in Russian]

3) Astaria T. Infrared thermography for thermo-fluid-dynamics / T. Astaria, G. M. Carlomagno. - Berlin : Springer-Verlag Berlin Heidelberg, 2013. - 226 p.

4) Strukturnaya diagnostika fakela $v$ real'nom vremeni kak osnova sozdaniya sovremennyh sistem upravleniya / P. YU. Hudyakov, I. A. Berg, N. A. Melkozyorov, B. P. ZHilkin // Sbornik dokladov VIII mezhdunarodnoj nauchno-tekhnicheskoj konferencii studentov, aspirantov i molodyh uchyonyh "Energiya-2013". - 2013. - 24-25 april. - P. 191-195. [in Russian]

5) Primenenie teplovizionnogo metoda dlya obnaruzheniya ochagov plamennogo goreniya / B. P. Zpilkin, N. S. Zajkov, A. Yu. Kisel'nikov, P. Yu. Hudyakov, S. G. Alekseev // Fire and Explosion Safety. - 2012. - Vol. 21, № 4. - PP. 38-40. [in Russian]

6) Berg I. A. Identification of Pulsating Combustion Modes of Gazeous Fuel / I. A. Berg, S. V. Porshnev, B. P. Zhilkin //AIP Conference Proceedings 1906. - 2017. - P. 070016.

7) Syzrancev V. N. Raschet prochnostnoj nadezhnosti izdelij na osnove metodov neparametricheskoj statistiki / V. N. Syzrancev, YA. P. Nevelev, S. L. Golofast. - Novosibirsk : Science, 2008. - 218 p. [in Russian]

8) Porshnev S. V., Koposov A. S. Programm library ES\&RP. - Federal registration certificate // Federal registration certificate № 2016614275 (Application № 2016611747 from 2 march of 2016. Issued 20 april of 2016).

9) Warnatz J. Concentration-, Pressure-, and Temperature-Dependence of the Flame Velocity in Hydrogen-Oxygen-Nitrogen Mixtures / J. Warnatz // Combustion Science and Technology. - 1981. - Vol. 26, Issue 5-6. - P. 203-213. 
10)Testing the null hypothesis of stationarity against the alternative of a unit root / D. Kwiatkowski, P. C. B. Phillips, P. Schmidt, Y. Shin // Journal of Econometrics. - 1992. - Vol. 54. P. 159-178.

11) Econometrics Toolbox Product Description [Electronic resource] / The MathWorks, Inc. // Matlab documentation. - Mode of access: https://www.mathworks.com/help/econ/product-description.html (date of accsess: 24.06.2019).

12) Berg I. A. Pulsating combustion: Theoretical and empirical substantiation of ecological effect / I. A. Berg, S. V. Porshnev, V. Y. Oshchepkova // AIP Conference Proceedings 1910. 2017. - P. 020010. 